\title{
How Can We Improve the Use of Established Therapies in PsA?
}

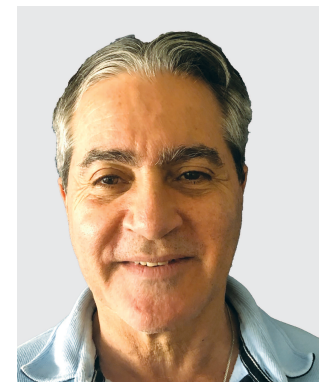

Just because nobody complains it doesn't mean all parachutes are perfect. - Benny Hill

While there has been an expansion in therapeutic targets and a renaissance of novel therapies in psoriatic arthritis (PsA) ${ }^{1}$, the efficacy and safety of tumor necrosis factor inhibitors (TNFi) in PsA have long been established ${ }^{2,3}$. With an influx of "cheaper" biosimilars attractive to regulators and reimbursers, it behooves rheumatologists spoiled with choice to maximize efficient use of readily accessible agents.

Immunogenicity due to anti-drug antibodies (ADAb) in TNFi therapy has been shown to affect drug levels and subsequently to affect clinical response in rheumatoid arthritis (RA) ${ }^{4}$ and inflammatory bowel disease ${ }^{5}$. In turn it affects safety, inducing infusion and injection site reactions (ISR), but the issue has been less well studied in patients with PsA.

Evidence from RA studies has shown that the combination of methotrexate (MTX) with TNFi reduces immunogenicity, significantly prolonging drug survival ${ }^{6}$. PsA data comparing monotherapy and combination therapy from Swedish and Consortium of Rheumatology Researchers of North America registries examining drug survival as a surrogate for persisting efficacy without major adverse effects that would lead to discontinuation have shown that in contrast to RA and with the exception of infliximab (IFX; a chimeric monoclonal antibody), the addition of MTX to either adalimumab (ADA) or etanercept (ETN) failed to improve drug survival ${ }^{7,8}$.

To complicate matters, measuring ADAb with precise sensitivity is not straightforward. Bridging ELISA can detect "free" ADAb but not antibodies bound in immune complexes with the treatment drug. Pretreating samples by acid dissociation significantly improves ADAb detection in the presence of the drug.

Menter, et $a l^{9}$ had previously demonstrated, in patients with plaque psoriasis, low-dose as-needed IFX therapy was associated with a higher immunogenicity $(46.2 \%)$ than the high-dose continuous treatment $(35.8 \%)$. Similar observations were also made in patients with Crohn disease.
What do we know specifically in PsA? Analyses from the study of golimumab (GOL) in PsA (GO-REVEAL) ${ }^{10}$ have shown that patients with higher ADAb levels had lower serum GOL concentrations, and that serum GOL concentrations appeared to decrease as peak ADAb titers increased across studies at Week 24 and Week 52. In addition, ADAb affected serum GOL concentrations when titers exceeded 1:100, and an effect on clinical response was observed only with the highest titers $(>1: 1000)$. The small number of ISR limited the ability to evaluate the effect of ADA titers on safety; however, $\mathrm{ADAb}+$ patients had neither severe nor serious ISR, and these did not lead to discontinuation ${ }^{8}$. Previous work has demonstrated that the majority of $\mathrm{ADAb}+$ patients had neutralizing antibodies. Many factors can influence immunogenicity findings, including sample handling, timing of collection, concomitant medications, and underlying disease.

Balsa, et al ${ }^{11}$ performed a systematic literature review of the comparative immunogenicity of biologic therapy and its clinical relevance in PsA and showed that ADAb rates varied widely among studies with biologic therapeutic agents in PsA, with the highest frequency in patients treated with ADA and IFX, intermediate frequency in patients treated with GOL and ustekinumab, and the lowest in patients treated with ETN and secukinumab. Concomitant use of MTX was associated with lower rates of ADAb. Patients with ADAb against ADA, IFX, and ustekinumab had significantly lower, sometimes undetectable, serum drug levels compared to those without $\mathrm{ADAb}^{8}$.

In this issue of The Journal, Jani, et al examined the effect of pharmacological "biomarkers," in this instance ADAb, in patients with PsA who were commencing biologic disease-modifying antirheumatic drugs (bDMARD) ${ }^{12}$. In particular their objectives were to identify:

- whether the presence of ADAb/drug levels predicted treatment response and disability in TNFi-treated patients with PsA;

- a drug level threshold for optimal therapeutic response;

- the factors associated with drug levels.

See Biomarkers, treatment response, and disability in PsA, page 1204

Personal non-commercial use only. The Journal of Rheumatology Copyright (C) 2020. All rights reserved. 
Biomarkers generally refer to objectively measurable characteristics that are indicators of biological processes rather than factors that affect efficacy and safety. The authors used 28-joint count Disease Activity Score (DAS28) with $\mathrm{C}$-reactive protein (CRP) as a measure of disease activity; this is less appropriate in PsA because it leaves out commonly affected joints such as distal interphalangeal joints, and joints in the feet and ankles. The Health Assessment Questionnaire (HAQ) was used as an indicator of disability and effect on quality of life but would have a considerable irreversible element in this cohort, with disease duration of 5 to 6 years and severity sufficient to require a bDMARD. ADAb was measured by radioimmunoassay and drug levels by ELISA.

Among the findings:

- Twenty percent of patients taking ADA had ADAb, while no patients using ETN had them. Given that ETN is a fusion protein, additional assays would have been needed to measure hinge/binding antibodies that affect drug clearance.

- Drug levels were associated with DAS28-CRP and inversely related with HAQ over 12 months.

- Change in DAS28-CRP was not independently associated with $\mathrm{ADAb}$ level.

- ADA concentrations of 4-8 $\mu \mathrm{g} / \mathrm{ml}$ were associated with optimal treatment response at 6 months, and concentration was inversely associated with ADAb and body mass index.

The study concluded that drug level testing in ADA-initiated patients with PsA may be useful in determining treatment response and disability at least over 12 months. Identification of a drug level threshold for optimal response may help tailor ADA therapy for patients with PsA in the future, with opportunities for serum concentrationguided dose tapering and more informed dosage adjustments in non- or partial responders.

PETER NASH ${ }^{(D)}$, MBBS(hons), FRACP Griffith University School of Medicine, Brisbane, Queensland,

Australia.

Address correspondence to Dr. P. Nash, Griffith University, Medicine, PO Box 308, Sunshine Coast, Nathan, Queensland 4111,

Australia.E-mail: drpnash@tpg.com.au

\section{REFERENCES}

1. Nash P. Psoriatic arthritis: novel targets add to a therapeutic renaissance. Lancet 2018;391:2187-9.
2. Coates LC, Kavanaugh A, Mease PJ, Soriano ER, Laura Acosta-Felquer M, Armstrong AW, et al. Group for Research and Assessment of Psoriasis and Psoriatic Arthritis 2015 Treatment Recommendations for Psoriatic Arthritis. Arthritis Rheumatol 2016;68:1060-71.

3. Gossec L, Smolen JS, Ramiro S, de Wit M, Cutolo M, Dougados M, et al. European League Against Rheumatism (EULAR) recommendations for the management of psoriatic arthritis with pharmacological therapies: 2015 update. Ann Rheum Dis 2016;75:499-510.

4. Bartelds G, Krieckaert C. Development of antidrug antibodies against adalimumab and association with disease activity and treatment failure during long-term follow-up. JAMA 2011;305:1460-8.

5. Hindryckx P, Novak G, Vande Casteele N, Khanna R, Laukens D, Jairath $\mathrm{V}$, et al. Incidence, prevention and management of anti-drug antibodies against therapeutic antibodies in inflammatory bowel disease: a practical overview. Drugs 2017;77:363-77.

6. Jani M, Barton A, Warren RB, Griffiths CE, Chinoy H. The role of DMARDs in reducing the immunogenicity of TNF inhibitors in chronic inflammatory diseases. Rheumatology 2014;53:213-22.

7. Fagerli KM, Lie E, van der Heijde D, Heiberg MS, Lexberg AS, $\mathrm{R} \emptyset \mathrm{devand} \mathrm{E}$, et al. The role of methotrexate co-medication in TNF-inhibitor treatment in patients with psoriatic arthritis: results from 440 patients included in the NOR-DMARD study. Ann Rheum Dis 2014;73:132-7.

8. Mease PJ, Collier DH, Saunders KC, Li G, Kremer JM, Greenberg JD. Comparative effectiveness of biologic monotherapy versus combination therapy for patients with psoriatic arthritis: results from the Corrona registry. RMD Open 2015;1:e000181.

9. Menter A, Papp KA, Gooderham M, Pariser DM, Augustin M, Kerdel FA, et al. Drug survival of biologic therapy in a large, disease-based registry of patients with psoriasis: results from the Psoriasis Longitudinal Assessment and Registry (PSOLAR). Eur Acad Dermatol Venereol 2016;30:1148-58.

10. Leu JH, Adedokun OJ, Gargano C, Hsia EC, Xu Z, Shankar G. Immunogenicity of golimumab and its clinical relevance in patients with rheumatoid arthritis, psoriatic arthritis and ankylosing spondylitis. Rheumatology 2019;58:441-6.

11. Balsa A, Lula S, Marshall L, Szczypa P, Aikman L. The comparative immunogenicity of biologic therapy and its clinical relevance in psoriatic arthritis: a systematic review of the literature. Expert Opin Biol Ther 2018;18:575-84.

12. Jani M, Chinoy H, Barton A. Association of pharmacological biomarkers with treatment response and long-term disability in patients with psoriatic arthritis: results from OUTPASS. J Rheumatol 2020;47:1204-8.

J Rheumatol 2020;47:1153-4; doi:10.3899/jrheum.190999 